\title{
Displacement Estimation of Prestressced Concrete Girder using Artificial Neural Network
}

\author{
Tae Min Kim, Hyun Su Moon, Moon Kyum Kim, Yun Mook Lim \\ Department of Civil \& Environmental Engineering, Yonsei University \\ 50 Yonsei-ro Seodaemun-gu, Seoul, Republic of Korea \\ taemin@yonsei.ac.kr; smilefou@naver.com; applymkk@yonsei.ac.kr; yunmook@yonsei.ac.kr
}

\section{Extended Abstract}

The purpose of the present study is to develop a method to derive the displacement from measured strain of several points on the structure with a back-propagation neural network. The structural displacement distribution gives us lots of information about the global behavior of structure. The intuitive information allows us not only to predict the load applied to the structure like weigh-in-motion system [1], but also to evaluate the condition of the structure. Especially, long-term displacement measurement provides a rationale to evaluate a degradation and aging of structure [2]. In addition to the mentioned advantages, displacement is superior to strain in terms of the characteristic that rigid motion can be tracked. The information is important when eccentricity of compression member which results in buckling failure or collision with other structures should be considered.

Furthermore, because the displacement may affect the human sensitivity, several specifications specify the maximum allowable displacement. Specifications for highway bridges in Korea also have a live load deflection check. This limitations also work to prevent the deterioration of wearing surfaces and to reduce the risk of potential impact to primary members by underpass traffic [3].

As described, though the displacement provides important information, it is not easy to measure it directly. For example, it is not always easy to place the measuring device appropriately, or the measuring cost is not always admissible. Unlike the displacement measurement, strain measurement is not very difficult. The apparatus can set up regardless of the location. In addition, it is possible to conduct real-time measurement. A Fiber Bragg grating (FBG) sensor can highlight the advantage. The FBG sensor allows us to measure the strain accurately, and it has a tolerance for the severe environment.

Since the strain measurement is easier than the displacement measurement, there have been several studies on developing the method to derive the displacement from the strain. Kang et al. [4] developed the model to derive the displacement response from the strain measurement by FBG sensor, however the model can be applied to one dimensional simple beam; therefore it cannot be applied to the real bridge. According to Euler-Bernoulli beam theory, the displacement can be obtained from the integration of the strain. The theory was developed on the assumption that normal to the neutral surface remain normal during the deformation and that deflections are small. However, the real structure does not necessarily satisfy these assumptions including bridges. Furthermore, integration constants should be determined from boundary conditions, but these conditions cannot be determined precisely in the real structure (e.g. perfect simple-support does not exist where the rotation is completely free).

In order to overcome the above described difficulty, the present research purposes to develop the method to derive displacement from measured strain of several points on the structure within a neural network framework. The method can be applied to the structure which does not satisfy the assumptions shown above. In addition, this method is applicable for very complex loading conditions. There exist several techniques in the neural network analysis. Among them, the present research uses multilayer neural network employing back-propagation algorithm, which is designed to reduce error between the actual output and the desired output of the network. In this research, full-scale 60-m PSC girder is employed to see the applicability. The displacement and strain values used here are obtained from experimental results and verified by finite element analysis (FEA). Using the FEA, various cases of loading conditions are generated and applied. The advantage of using the FEA is that displacement which is difficult to obtain in the real measurement is easily derived from calculation; 
therefore it is easier to validate the method developed here. Then, the validity of the developed neural network model is confirmed. The displacement values derived from the developed neural network model was compared with those derived from FEA.

\section{References}

[1] F. Moses, "Weigh-in-motion system using instrumented bridges," J. Transportation Eng., vol. 105, no. 3, pp. 233249, 1979.

[2] W. Kim, J. A. Laman, "Numerical analysis method for long-term behaviour of integral abutment bridges," Eng Struct., vol. 32, no. 8, pp. 2247-2257, 2010.

[3] J. J. Zhao, D. E. Tonias. Bridge Engineering, 3rd ed, McGraw-Hill, 2012.

[4] L.-H. Kang, D.-K. Kim, J.-H. Han, "Estimation of dynamic structural displacements using fiber Bragg grating strain sensors," J. Sound Vib., vol. 305, no. 3, pp. 534-542, 2007. 